\title{
Females with Impaired Ovarian Function Could Be Vulnerable to Environmental Pollutants: Identification via Next-Generation Sequencing of the Vaginal Microbiome
}

\section{Seongmin Kim}

CHA Ilsan Medical Center https://orcid.org/0000-0003-2541-8879

Se Hee Lee

Korea University Graduate School

Kyung Jin Min

Korea University College of Medicine

\section{Sanghoon Lee}

Korea University College of Medicine

Jin Hwa Hong

Korea University College of Medicine

Jae Yun Song ( $\square$ yuni105@korea.ac.kr)

Korea University College of Medicine

Jae Kwan Lee

Korea University College of Medicine

\section{Nak Woo Lee}

Korea University College of Medicine

Eun II Lee

Korea University College of Medicine

Research article

Keywords: vaginal microbiome, premature ovarian insufficiency, environmental pollutants

Posted Date: December 10th, 2019

DOI: https://doi.org/10.21203/rs.2.18527/v1

License: (c) (1) This work is licensed under a Creative Commons Attribution 4.0 International License.

Read Full License 
Version of Record: A version of this preprint was published at Journal of Obstetrics and Gynaecology on January 7th, 2022. See the published version at https://doi.org/10.1080/01443615.2021.2006162. 


\section{Abstract}

Background: The vaginal microbiome has been widely investigated because of its utility in the diagnosis of vaginitis and its connection to various conditions. However, its relationship with impaired ovarian function and the influence of environmental pollutants have not been evaluated. Here, we conducted a next-generation sequencing study of the vaginal microbiome in young females with normal and decreased ovarian function and analyzed its correlation with various environmental pollutants.

Methods: Vaginal swabs were collected from 92 individuals (22 with impaired ovarian function and 70 with normal function), and the vaginal microbiome was analyzed using next-generation sequencing. The 16S rDNA sequences were assembled by Fast Length Adjustment of SHort reads (FLASH) and clustered by Operational Taxonomic Units (OTUs). Diversity analysis was performed using Quantitative Insights Into Microbial Ecology (QIIME). Laboratory findings, including hormonal status were measured.

Results: Most characteristics of the two groups were similar, except that the impaired function group showed lower AMH and higher FSH ( $p<0.01$ and $p=0.04$, respectively). Among the most abundant taxa, several species differed; however, only two species showed significant differences: Propionibacterium acnes and Prevotella copri. The heat map did not show significant differences between the groups. Canonical correlation analysis revealed that more environmental pollutants were related to changes in the vaginal microbiome in the impaired ovarian function group than in the normal group.

Conclusion: The vaginal microbiomes in young women with decreased ovarian function tended to be more sensitive to various environmental pollutants, especially volatile organic compounds (VOCs). Therefore, females with impaired ovarian function could be more vulnerable to VOCs.

\section{Background}

The microbiome, the collective genetic material from a microbial community in a particular environment, varies according to body site and individual (1). Increasing evidence has demonstrated that the microbiome has a considerable effect on host metabolism and the response to various diseases (2). Although the techniques used for analysis of microbiomes are continuously improving, from culturedependent methods to DNA and RNA sequencing (3), our knowledge of the role of the microbiome in human health and disease is still limited.

The vaginal microbiome has been widely investigated because of its value in the diagnosis of vaginitis and association with other conditions (4-10). Our knowledge of the vaginal microbiota has been extended by the development of molecular approaches based on 16S rRNA gene cloning and sequencing, which has allowed the identification of taxa that were not previously detected by conventional culture methods. A distinct bacterial community, with a low abundance of Lactobacillus, was shown to be associated with vaginal atrophy in menopausal women (11), implying that the composition of the vaginal microbiome could be related to menopausal conditions. Premature ovarian insufficiency (POI), which is the depletion or dysfunction of ovaries with menopause or ovarian failure before age 40 , is a relatively 
poorly understood condition with a various etiologies (12). Despite ongoing efforts, the advancements on our understanding and treatment of $\mathrm{POI}$ have been minimal, and clinicians remain frustrated when managing patients with $\mathrm{POI}$. The proposed etiologies of POI are thought to be genetic, iatrogenic, metabolic, infectious, or autoimmune (13). Recently, there has been some indication that environmental factors contribute to the onset of POI, by acting as major determinants of ovarian reserve (14). However, the relationship between environmental factors and POI has not been evaluated.

In this study, we investigated the association of the vaginal microbiome with environmental factors and ovarian damage by evaluating the vaginal microbiome in patients with impaired ovarian function using next-generation sequencing and its association with environmental pollutants.

\section{Materials And Methods}

Study population. The individuals enrolled in this study were part of the "Environmental Health Action Program" of the Korean Ministry of Environment. This program was designed to build a cohort of patients with premature ovarian failure (POF) and to determine the mechanism of POF using animal models. The study was approved by ethical committee of Korea University Medical Center Anam Hospital (No. 2016AN0251) and was performed in accordance with relevant guidelines and regulations of the committee. The participants were recruited voluntarily via an online survey system or at an outpatient clinic in multiple institutions from March 2016 to January 2017. Informed consent forms were obtained from all participants for the use of human-derived materials and their personal information. All women were 30-45 years old and had not taken any antibiotics in the past 30 days.

Specimen acquisition and cohort composition. The following samples were obtained from all participants: serum $(10 \mathrm{ml})$, urine $(40 \mathrm{ml})$, and vaginal swabs. The serum analysis included hormone levels, thyroid function, prolactin, lipid profiles, and other basic laboratory findings (Table 1). All participants were administered human papilloma virus (HPV) and Pap tests, and vaginal swabs were also obtained for next-generation sequencing (NGS) of the vaginal microbiome. The urine samples were sent to the Department of Preventive Medicine, where the concentrations of various environmental pollutants were determined. The populations were divided in three groups according to hormonal status (Table 2). However, in this study, patients with POI and participants with decreased AMH were combined into one group, and labeled as the "impaired ovarian function" (IO) group. 
Table 1

Patient samples collected and analyses performed on them

\section{Specimen Analyzed components}

Serum $\quad$ CBC, electrolytes, liver function tests (AST, ALT, bilirubin, ALP, protein, and albumin), lipid profile (TG, HDL, LDL, TC), thyroid functional tests (TSH and free T4), and hormones ( $\mathrm{FSH}, \mathrm{E} 2$, progesterone, $\mathrm{AMH}$, and prolactin)

Urine

Phthalates (MEHHP and MEOHP), phenols (BPA, MP, EP, PP, and 24DCP), cotinine, VOCs (ttMA, BMA, PGA, MA, and MHA), 1-OHP, and 2-NAP

Vaginal Next generation sequencing of the vaginal microbiome smear

1-OHP, 1-hydroxypyrene; 2-NAP, 2-naphthol, 24DCP, 2,4-dichlorophenol; ALT, alanine amino-transferase; $\mathrm{AMH}$, anti-Müllerian hormone; AST, aspartate aminotransferase; BMA, benzyl mercapturic acid; BPA, 2,2-Bis(4-hydroxyphenyl)propane(also known as bisphenol A); CBC, complete blood cell count; E2, estradiol; EP, ethyl phenol; FSH, follicular stimulating hormone; HDL, high density lipoprotein; LDL, low density lipoprotein; MA, mandelic acid; MEHHP, mono 2-ethyl-5-hydroxyhexyl phthalate; MEOHP, mono 2-ethyl-5-oxohexyl phthalate; MHA, methyl hippuric acid; MP, methyl phenol; PGA, phenyl glyoxylic acid; PP, propyl phenol; TC, total cholesterol; TG, triglyceride; TSH, thyroid stimulating hormone; ttMA, trans,trans-muconic acid; VOCs, volatile organic compounds

Table 2

Patient groups.

\section{Group Definition}

$\mathrm{POI}$

Borderline†

Normal
Amenorrhea for $>1$ year, $\mathrm{FSH} \geq 40$.

Menstruating, but with decreased $\mathrm{AMH}$

(Value as high as 2 years older than actual age)

All study participants were females aged $35-40$ years.

$\mathrm{AMH}$, anti-Müllerian hormone; $\mathrm{FSH}$, follicle stimulating hormone; $\mathrm{POI}$, premature ovarian insufficiency

†These two groups were combined in the impaired ovarian function (IO) group.

Analysis of toxic chemical metabolites in urine. Urine was stored $-70{ }^{\circ} \mathrm{C}$ until analysis, and during the analysis, contact with plastic was avoided. Fifteen metabolites were analyzed by Smartive Corporation (Seoul, Republic of Korea). Polycyclic aromatic hydrocarbons (PAHs; (1-hydroxypyrene and 2hydroxynaphthalene) and cotinine (COT) metabolites were analyzed using a gas chromatograph-mass spectrometer (GC-MS, Clarus 680T, Perkin Elmer, USA).

The following compounds were analyzed by using a high performance liquid chromatography-triple tandem mass detector (HPLC-MS/MS, 6410B, Agilent, USA): phthalate metabolites [mono(2-ethyl-5hydroxyhexyl) phthalate (MEHHP) and mono(2-ethyl-5-oxohexyl) phthalate (MEOHP)], environmental 
phenols [bisphenol A (BPA), methyl paraben (MP), ethyl paraben (EP), propyl paraben (PP), and 2,4dichlorophenol (24DCP)], volatile organic compounds [VOCs: trans, trans-muconic acid (ttMA), benzyl mercapturic acid (BMA), phenyl glyoxylic acid (PGA), and o,m,p-methyl hippuric acid (MHA), mandelic acid (MA)].

MiSeq-metagenomic sequencing. Genomic DNA was extracted from the vaginal swabs using the Power Soil DNA Isolation Kit (MOBIO, Carlsbad, CA, USA). The 16S rRNA genes were amplified according to the Illumina 16S Metagenomic Sequencing Library protocols as follows. Universal primers (16S Amplicon PCR Forward 5'-TCGTCGGCAGCGTCAGATGTGTATAAGAGACAGCCTACGGGNGGCWGCAG and 16S Amplicon PCR Reverse 5'-

GTCTCGTGGGCTCGGAGATGTGTATAAGAGACAGGACTACHVGGGTATCTAATCC) were used for PCR amplification of the V3-V4 hypervariable regions of the 16S rRNA gene from $12.5 \mathrm{ng}$ of the genomic DNA prepared from each sample. A subsequent limited-cycle amplification step was performed to add multiplexing indices and Illumina sequencing adapters. The final products were normalized and pooled using PicoGreen, and the size of libraries was verified using the LabChip GX HT DNA High Sensitivity Kit (PerkinElmer, MA, USA). We used the MiSeq ${ }^{\text {TM }}$ platform (Illumina, San Diego, CA, USA) at Macrogen, Inc. (Seoul, Korea) for sequencing according to the manufacturer's instructions.

Operational taxonomic unit analysis. The sequence of each operational taxonomic unit (OTU) was used as a query against the NCBI reference 16 S Microbial database with BLASTN (v2.4.0) to obtain a taxonomic assignment by identifying the most similar species. Microbial community comparisons were performed by using QIIME (v1.8).

Canonical correlation analysis. The results of the urine analysis of 15 metabolites and the vaginal microbial population were subjected to canonical correlation analysis, which was conducted at the species level using R ( $v$ 3.1.2R).

\section{Results}

Characteristics of study participants. A total of 92 samples were obtained from the study participants. Analysis of hormonal levels revealed that of the 22 patients in the 10 group, five had definite POF, and the other 17 patients had impaired ovarian function with low AMH levels. The clinical characteristics and blood analysis results are shown in Table 3. Most variables were similar between the two groups, except for follicular stimulating hormone and anti-Müllerian hormone levels. 
Table 3

Clinical characteristics of the study participants.

\begin{tabular}{|c|c|c|c|c|}
\hline & & $\begin{array}{l}\text { Normal group } \\
(n=70)\end{array}$ & $\begin{array}{l}10 \text { group } \\
(n=22)\end{array}$ & p-value \\
\hline \multicolumn{2}{|l|}{ Age } & 33.8 & 33.9 & 0.87 \\
\hline \multicolumn{2}{|l|}{ BMI } & 23.15 & 23.07 & 0.47 \\
\hline \multirow[t]{3}{*}{ Parity } & 0 & 26 & 8 & \multirow[t]{3}{*}{0.79} \\
\hline & 1 & 34 & 12 & \\
\hline & 2 or more & 10 & 2 & \\
\hline \multicolumn{2}{|c|}{ History of ovarian surgery } & 8 & 2 & 0.76 \\
\hline \multicolumn{2}{|l|}{ AMH } & 5.07 & 0.71 & $<0.01$ \\
\hline \multicolumn{2}{|l|}{$\mathrm{FSH}$} & 3.96 & 15.28 & 0.01 \\
\hline \multicolumn{2}{|l|}{ E2 } & 77.81 & 76.81 & 0.58 \\
\hline \multicolumn{2}{|c|}{ Prolactin } & 10.82 & 8.85 & 0.20 \\
\hline \multicolumn{2}{|c|}{ Progesterone } & 5.11 & 7.55 & 0.26 \\
\hline \multicolumn{2}{|l|}{ TSH } & 2.61 & 2.67 & 0.75 \\
\hline \multicolumn{2}{|c|}{ Free T4 } & 1.28 & 1.21 & 0.08 \\
\hline \multicolumn{2}{|c|}{ Protein } & 7.31 & 7.33 & 0.86 \\
\hline \multicolumn{2}{|c|}{ Albumin } & 4.51 & 4.44 & 0.39 \\
\hline \multicolumn{2}{|c|}{ Total cholesterol } & 178.7 & 179.9 & 0.86 \\
\hline \multicolumn{2}{|c|}{ Direct bilirubin } & 0.17 & 0.11 & 0.33 \\
\hline \multicolumn{2}{|l|}{ AST } & 20.62 & 23.20 & 0.58 \\
\hline \multicolumn{2}{|l|}{ ALT } & 16.42 & 19.02 & 0.23 \\
\hline \multicolumn{2}{|c|}{ Hemoglobin } & 12.96 & 12.91 & 0.25 \\
\hline \multicolumn{2}{|c|}{ Platelets } & 266.4 & 270.0 & 0.81 \\
\hline \multicolumn{2}{|l|}{ WBC } & 6.13 & 6.43 & 0.41 \\
\hline \multicolumn{5}{|c|}{$\begin{array}{l}\text { ALT, alanine amino-transferase; AMH, anti-Müllerian hormone; AST, aspartate aminotransferase; E2, } \\
\text { estradiol; FSH, follicle stimulating hormone; HDL, high density lipoprotein; LDL, low density } \\
\text { lipoprotein; TSH, thyroid stimulating hormone; WBC, white blood cell }\end{array}$} \\
\hline
\end{tabular}


Next-generation sequencing of the vaginal microbiome. A total of 937 species were identified in the analysis of the vaginal microbiome, and 24 dominant species were selected for further analysis (Table 4). The samples had 56,338-147,588 read counts, and > 89.06\% had a PHRED (Q) score above 30, indicating a 1:1000 probability of an incorrect base call. Taxonomic abundance showed that lactobacilli and enterococci were main taxa in the normal group, but that the species tended to be more heterogeneous in the IO group (Fig. 1). A heat map was drawn using the taxonomy information, which showed that the two groups were not different from each other (Fig. 2). The proportions of each species were compared between the two groups, and only two species (Propionibacterium acnes and Prevotella copri) were significantly more abundant in the POI group (Fig. 3). The 3-D plot and functional analysis did not reveal a difference between the groups (Fig. 4, 5). 
Table 4

The 24 most dominant species identified in the vaginal microbiome, which were selected for further analysis..

\begin{tabular}{|l|}
\hline Species \\
\hline Lactobacillus crispatus \\
\hline Lactobacillus iners \\
\hline Lactobacillus paracasei \\
\hline Lactobacillus sakei \\
\hline Pseudomonas azotoformans \\
\hline Pseudomonas stutzeri \\
\hline Pseudomonas geniculata \\
\hline Prevotella amnii \\
\hline Prevotella copri \\
\hline Bacillus atrophaeus \\
\hline Bacillus nanhaiisediminis \\
\hline Propionibacterium acnes \\
\hline Gardnerella vaginalis \\
\hline Bacteroides vulgatus \\
\hline Parabacteroides goldsteinii \\
\hline Gemella bergeri \\
\hline Staphylococcus epidermidis \\
\hline Enterococcus hirae \\
\hline Weissella koreensis \\
\hline Streptococcus dentisani \\
\hline Megasphaera cerevisiae \\
\hline Sneathia sanguinegens \\
\hline Cupriavidus necator \\
\hline Moraxella osloensis \\
\hline
\end{tabular}

Page 9/21 
Table 5

Average amounts of the tested pollutants and their correlation with vaginal microbiome composition by canonical correlation analysis.

\begin{tabular}{|c|c|c|c|c|}
\hline & \multicolumn{2}{|l|}{ Normal } & \multicolumn{2}{|l|}{10} \\
\hline & $\mathrm{R}^{2}$ & p-value & $\mathrm{R}^{2}$ & p-value \\
\hline MEHHP & 0.00708 & 0.894 & 0.074706 & $0.025^{\star}$ \\
\hline MEOHP & 0.030157 & $0.022^{\star}$ & 0.0427908 & 0.259 \\
\hline BPA & 0.0089376 & 0.778 & 0.0306876 & 0.567 \\
\hline MP & 0.0110904 & 0.64 & 0.0840278 & $0.019 *$ \\
\hline EP & 0.054795 & $0.001^{*}$ & 0.0326023 & 0.52 \\
\hline PP & 0.0054108 & 0.918 & 0.0555429 & 0.109 \\
\hline @24DCP & 0.0142214 & 0.424 & 0.0263452 & 0.679 \\
\hline COT & 0.011136 & 0.622 & 0.0496253 & 0.167 \\
\hline ttMA & 0.0070096 & 0.914 & 0.0508416 & 0.146 \\
\hline BMA & 0.0106694 & 0.673 & 0.0713918 & $0.032^{\star}$ \\
\hline PGA & 0.0199757 & 0.163 & 0.0306561 & 0.564 \\
\hline MA & 0.0194256 & 0.178 & 0.0662586 & 0.052 \\
\hline MHA & 0.0120881 & 0.533 & 0.0723369 & $0.026 *$ \\
\hline 1-OHP & 0.0144537 & 0.374 & 0.0399658 & 0.373 \\
\hline 2-NAP & 0.0103992 & 0.674 & 0.0648005 & 0.062 \\
\hline \multicolumn{5}{|c|}{$\begin{array}{l}\text { 1-OHP, 1-hydroxypyrene; 2-NAP, 2-naphthol, 24DCP, 2,4-dichlorophenol; BMA, benzyl mercapturic acic } \\
\text { BPA, 2,2-Bis(4-hydroxyphenyl) propane(also known as bisphenol A); EP, ethyl phenol; MA, mandelic } \\
\text { acid; MEHHP, mono 2-ethyl-5-hydroxyhexyl phthalate; MEOHP, mono 2-ethyl-5-oxohexyl phthalate; } \\
\text { MHA, methyl hippuric acid; MP, methyl phenol; PGA, phenyl glyoxylic acid; PP, propyl phenol; ttMA, } \\
\text { trans,trans-muconic acid. }\end{array}$} \\
\hline *: p-value & & & & \\
\hline
\end{tabular}

Correlations with environmental pollutants. Urine samples were analyzed for 15 pollutants. The average amount of each pollutant and its correlation with the composition of the vaginal microbiome as determined using CCA are shown in Table 3. In the normal group, MEOHP and EP levels were significantly correlated with vaginal microbiome status, with a low $R^{2}$ value, while four other pollutants were related to species composition, showing a relatively higher $\mathrm{R}^{2}$ value in the POI group (Fig. 6). MA and 2-NAP were 
not significantly related to the composition of the vaginal microbiome, but they showed a tendency towards sensitivity in the IO group, with a higher $\mathrm{R}^{2}$ value than the normal group.

\section{Discussion}

The vaginal microbiome reflects not only the bacterial composition in the vagina but also the general condition of the host, including hormonal status and immunity $(15,16)$. Although most previous studies investigated the relationship between vaginal microbiome and health using animal models, a few human studies have examined the epidemiology of the vaginal microbiome and its relationship with general health. Only one study observed that the concentration of phthalate in human urine was associated with a lower antral follicle count (17).

In this study, the change in the composition of the vaginal microbiome in young females with impaired ovarian function was more related to environmental pollutants than the change observed in the healthy group. To our knowledge, this is the first report to investigate the influence of environmental pollutants on the vaginal microbiome in young females with decreased ovarian function. Although the square correlation was low for the majority of tested pollutants, the relevance was higher in the IO group than the normal healthy group, and a larger number of pollutants showed a significant relationship in the IO group. Although this does not prove that these pollutants are a causative factor of ovarian dysfunction, it implies that such pollutants could have a greater effect on the composition of the vaginal microbiome in this population; thus, young women with impaired ovarian function could be more vulnerable to these pollutants. This could be an important finding, especially given the prevalence of pollutants in this modern age.

As mentioned above, the canonical analysis showed that two environmental pollutants were correlated with the vaginal microbiome in the normal group, and that four were correlated in the 10 group. MEOHP and MEEHP are phthalates that are used as plasticizers to increase the flexibility and transparency of plastics; they are also used to make polyvinyl chloride (PVC) softer (18). EP and MP are phenols that are often included in sterilizers or deodorizers (19). People are frequently exposed to these chemicals in everyday life during their daily activities, and these pollutants were related to the vaginal microbiome in both groups.

BMA, MHA, and MA showed a tendency to affect the vaginal microbiome only in the IO group. These substances are VOCs, which are a diverse group of carbon-based chemicals that are volatile at room temperature. Sources of VOCs include vehicles, gas evaporation, and industrial processes (20); thus, VOCs are a considerable cause of air pollution, especially fine dust (21). Although we cannot conclude that these VOCs cause ovarian damage, the results of the CCA at least imply that VOCs could alter the vaginal condition in young females with impaired ovarian function. This finding should not be overlooked, because it could be an important issue given the current environment in South Korea, as urbanization, industrialization, and the activities of neighboring countries are worsening air pollution, which is an important risk factor for human diseases (22). Moreover, VOCs are identified in components 
of disposable sanitary pads (23). This is also a big issue in recent South Korea. All the participants of this study are at the age of users of the pads, so using disposable pads could cause harmful effect on vaginal condition in individuals with impaired ovarian function. More robust studies may confirm the relationship between VOCs and impaired ovarian function, and this information could be useful for counselling females with decreased function.

The most abundant species in the vaginal microbiome was Lactobacillus, which is consistent with previous studies (24-26). A healthy vagina is usually dominated by Lactobacillus species, as there are several mechanisms that inhibit its colonization by pathogens (27). In this study, there was no remarkable difference in the composition of the vaginal microbiome between the normal and impaired ovarian function groups. Only two species showed a significant difference in abundance in the IO group; however, their role is unclear. Brotman et al. evaluated the association of the vaginal microbiome with menopausal status and vaginal atrophy (11). They found that there was a distinct bacterial community with a low abundance of Lactobacillus in patients with vaginal atrophy, but there was no relationship in menopausal women without atrophy. The role of estrogen therapy was thought to be important. Hormone replacement therapy is effective for vaginal atrophy in menopausal women and helps maintain the abundance of Lactobacillus with maintenance of Lactobacillus dominance $(28,29)$. In our study, all patients with POF were receiving estrogen supplementation; therefore, the vaginal conditions may be not that different from those of the normal healthy group, as the use of hormone therapy might mask the influence of environmental pollutants. Therefore, further analysis is necessary to confirm the exact association.

There are a few limitations to this study. First, we were not able to obtain any information about presence of vaginitis or any other underlying disease. The existence of vaginitis or vaginal atrophy could be related to the differences observed in the vaginal microbiome (30). However, we retrospectively reviewed the medical records of all participants, and there were only three women suspicious of vulvovaginitis on physical examination (two in the normal group and one in the 10 group). This low incidence should not have caused a difference in the whole study population. Second, the number of patients with POF in this study was small, as most of the participants in the IO group were still menstruating and had some reproductive function, even though their AMH levels were low. Therefore, additional investigations including more patients with POF are necessary to definitively determine the influence of pollutants on the vaginal microbiome between women with normal ovarian function and patients with POF.

However, this study has important implications in terms of the unique analytic methods used to infer the effects of environmental pollutants on the vaginal microbiome depending on ovarian function. To our knowledge, this is the first evaluation of this association using next-generation sequencing. Previous studies investigated the association of the vaginal microbiome with menopause (31), perinatal outcome (32), preterm birth (33), sexually transmitted diseases (34), vaginitis (30), HPV (35), and urinary tract infections (36). No study has evaluated the relationship of the vaginal microbiome with environmental pollutants in young females with impaired ovarian function. A possible susceptibility of this group to 
VOCs was revealed by our study. Therefore, additional studies should be conducted to confirm the underlying mechanism.

\section{Conclusion}

The vaginal microbiome in young women with decreased ovarian function showed a tendency to be more sensitive to various environmental pollutants, especially VOCs, when compared to the microbiome of females with normal ovarian function. This implies a considerable association between impaired ovarian function and air pollution, and VOCs might be a hazard to females with diminished ovarian function. This finding could be helpful when counselling patients with POI.

\section{List Of Abbreviations}

24DCP; 2,4-dichlorophenol,

BMA; benzyl mercapturic acid,

BPA; bisphenol A,

COT; cotinine,

EP; ethyl paraben,

HPV; human papilloma virus,

IO; impaired ovarian function ,

MA; mandelic acid,

MHA; o,m,p-methyl hippuric acid,

MEHHP; mono(2-ethyl-5-hydroxyhexyl) phthalate,

MEOHP; mono(2-ethyl-5-oxohexyl) phthalate,

MP; methyl paraben,

NGS; next-generation sequencing,

OTU; operational taxonomic unit,

PAH; Polycyclic aromatic hydrocarbon,

PGA; phenyl glyoxylic acid,

POF; premature ovarian failure, 
POI; Premature ovarian insufficiency,

PP; propyl paraben,

PVC; polyvinyl chloride

ttMA; trans, trans-muconic acid,

VOC; volatile organic compound,

\section{Declarations}

Ethics approval and consent to participate The study was approved by ethical committee of Korea University Medical Center Anam Hospital (No. 2016AN0251).

Consent for publication All participants provided written informed consent for biological studies.

Data availability statement The datasets generated during and/or analysed during the current study are available from the corresponding author on reasonable request.

Competing interests The authors declare no competing interests.

Funding This study was granted by the Korea Ministry of Environment (MOE) as 'The Environmental Health Action Program' (2016001360007).

\section{Author's contribution}

SK: acquisition of data, data analysis, writing the manuscript.

SHL: data interpretation, revising the manuscript.

KJM: acquisition of data

SL: acquisition of data

$\mathrm{JHH}$ : acquisition of data

JKL: acquisition of data

NWL: acquisition of data

EIL: data analysis, conception

JYS: conception and design, revising the manuscript, final approval of manuscript

Acknowledgements Not applicable. 


\section{References}

1. Group NHW, Peterson J, Garges S, Giovanni M, Mclnnes P, Wang L, et al. The NIH Human Microbiome Project. Genome Res. 2009;19(12):2317-23.

2. Turnbaugh PJ, Ley RE, Hamady M, Fraser-Liggett CM, Knight R, Gordon JI. The human microbiome project. Nature. 2007;449(7164):804-10.

3. Relman DA. The Human Microbiome and the Future Practice of Medicine. JAMA. 2015;314(11):11278.

4. Wang J, Li C, Nesengani LT, Gong Y, Zhang S, Lu W. Characterization of vaginal microbiota of endometritis and healthy sows using high-throughput pyrosequencing of 16S rRNA gene. Microb Pathog. 2017;111:325-30.

5. Di Paola M, Sani C, Clemente AM, lossa A, Perissi E, Castronovo G, et al. Characterization of cervicovaginal microbiota in women developing persistent high-risk Human Papillomavirus infection. Sci Rep. 2017;7(1):10200.

6. Chaban B, Links MG, Jayaprakash TP, Wagner EC, Bourque DK, Lohn Z, et al. Characterization of the vaginal microbiota of healthy Canadian women through the menstrual cycle. Microbiome. 2014;2:23.

7. Muzny CA, Sunesara IR, Kumar R, Mena LA, Griswold ME, Martin DH, et al. Characterization of the vaginal microbiota among sexual risk behavior groups of women with bacterial vaginosis. PLoS One. 2013;8(11):e80254.

8. Lambert JA, Kalra A, Dodge CT, John S, Sobel JD, Akins RA. Novel PCR-based methods enhance characterization of vaginal microbiota in a bacterial vaginosis patient before and after treatment. Appl Environ Microbiol. 2013;79(13):4181-5.

9. Shi Y, Chen L, Tong J, Xu C. Preliminary characterization of vaginal microbiota in healthy Chinese women using cultivation-independent methods. J Obstet Gynaecol Res. 2009;35(3):525-32.

10. Thies FL, Konig W, Konig B. Rapid characterization of the normal and disturbed vaginal microbiota by application of 16S rRNA gene terminal RFLP fingerprinting. J Med Microbiol. 2007;56(Pt 6):75561.

11. Brotman RM, Shardell MD, Gajer P, Fadrosh D, Chang K, Silver MI, et al. Association between the vaginal microbiota, menopause status, and signs of vulvovaginal atrophy. Menopause. 2014;21(5):450-8.

12. Torrealday S, Kodaman P, Pal L. Premature Ovarian Insufficiency - an update on recent advances in understanding and management. F1000Res. 2017;6:2069.

13. Vabre P, Gatimel N, Moreau J, Gayrard V, Picard-Hagen N, Parinaud J, et al. Environmental pollutants, a possible etiology for premature ovarian insufficiency: a narrative review of animal and human data. Environ Health. 2017;16(1):37.

14. Richardson MC, Guo M, Fauser BC, Macklon NS. Environmental and developmental origins of ovarian reserve. Hum Reprod Update. 2014;20(3):353-69. 
15. Jespers V, Kyongo J, Joseph S, Hardy L, Cools P, Crucitti T, et al. A longitudinal analysis of the vaginal microbiota and vaginal immune mediators in women from sub-Saharan Africa. Sci Rep. 2017;7(1):11974.

16. Moncla BJ, Chappell CA, Debo BM, Meyn LA. The Effects of Hormones and Vaginal Microflora on the Glycome of the Female Genital Tract: Cervical-Vaginal Fluid. PLoS One. 2016;11(7):e0158687.

17. Messerlian C, Souter I, Gaskins AJ, Williams PL, Ford JB, Chiu YH, et al. Urinary phthalate metabolites and ovarian reserve among women seeking infertility care. Hum Reprod. 2016;31(1):75-83.

18. Wallner P, Kundi M, Hohenblum P, Scharf S, Hutter HP. Phthalate Metabolites, Consumer Habits and Health Effects. Int J Environ Res Public Health. 2016;13(7).

19. Aker AM, Watkins DJ, Johns LE, Ferguson KK, Soldin OP, Anzalota Del Toro LV, et al. Phenols and parabens in relation to reproductive and thyroid hormones in pregnant women. Environ Res. 2016;151:30-7.

20. Okada Y, Nakagoshi A, Tsurukawa M, Matsumura C, Eiho J, Nakano T. Environmental risk assessment and concentration trend of atmospheric volatile organic compounds in Hyogo Prefecture, Japan. Environ Sci Pollut Res Int. 2012;19(1):201-13.

21. Jones AP. Dust evolution, a global view: III. Core/mantle grains, organic nano-globules, comets and surface chemistry. R Soc Open Sci. 2016;3(12):160224.

22. Leem JH, Kim ST, Kim HC. Public-health impact of outdoor air pollution for 2(nd) air pollution management policy in Seoul metropolitan area, Korea. Ann Occup Environ Med. 2015;27:7.

23. Bae J, Kwon H, Kim J. Safety Evaluation of Absorbent Hygiene Pads: A Review on Assessment Framework and Test Methods. Sustainability. 10, 4146, doi:10.3390/su10114146 (2018)

24. Huang B, Fettweis JM, Brooks JP, Jefferson KK, Buck GA. The changing landscape of the vaginal microbiome. Clin Lab Med. 2014;34(4):747-61.

25. Gajer P, Brotman RM, Bai G, Sakamoto J, Schutte UM, Zhong X, et al. Temporal dynamics of the human vaginal microbiota. Sci Transl Med. 2012;4(132):132ra52.

26. Zhou X, Bent SJ, Schneider MG, Davis CC, Islam MR, Forney LJ. Characterization of vaginal microbial communities in adult healthy women using cultivation-independent methods. Microbiology. 2004;150(Pt 8):2565-73.

27. Ravel J, Gajer P, Abdo Z, Schneider GM, Koenig SS, McCulle SL, et al. Vaginal microbiome of reproductive-age women. Proc Natl Acad Sci U S A. 2011;108 Suppl 1:4680-7.

28. Heinemann C, Reid G. Vaginal microbial diversity among postmenopausal women with and without hormone replacement therapy. Can J Microbiol. 2005;51(9):777-81.

29. Ozkinay E, Terek MC, Yayci M, Kaiser R, Grob P, Tuncay G. The effectiveness of live lactobacilli in combination with low dose oestriol (Gynoflor) to restore the vaginal flora after treatment of vaginal infections. BJOG. 2005;112(2):234-40.

30. Hong KH, Hong SK, Cho SI, Ra E, Han KH, Kang SB, et al. Analysis of the Vaginal Microbiome by NextGeneration Sequencing and Evaluation of its Performance as a Clinical Diagnostic Tool in Vaginitis. 
Ann Lab Med. 2016;36(5):441-9.

31. Muhleisen AL, Herbst-Kralovetz MM. Menopause and the vaginal microbiome. Maturitas. 2016;91:4250.

32. Prince AL, Chu DM, Seferovic MD, Antony KM, Ma J, Aagaard KM. The perinatal microbiome and pregnancy: moving beyond the vaginal microbiome. Cold Spring Harb Perspect Med. 2015;5(6).

33. Stout MJ, Zhou Y, Wylie KM, Tarr PI, Macones GA, Tuuli MG. Early pregnancy vaginal microbiome trends and preterm birth. Am J Obstet Gynecol. 2017;217(3):356 e1- e18.

34. Lewis FM, Bernstein KT, Aral SO. Vaginal Microbiome and Its Relationship to Behavior, Sexual Health, and Sexually Transmitted Diseases. Obstet Gynecol. 2017;129(4):643-54.

35. Mitra A, Maclntyre DA, Marchesi JR, Lee YS, Bennett PR, Kyrgiou M. The vaginal microbiota, human papillomavirus infection and cervical intraepithelial neoplasia: what do we know and where are we going next? Microbiome. 2016;4(1):58.

36. Stapleton AE. The Vaginal Microbiota and Urinary Tract Infection. Microbiol Spectr. 2016;4(6).

\section{Figures}

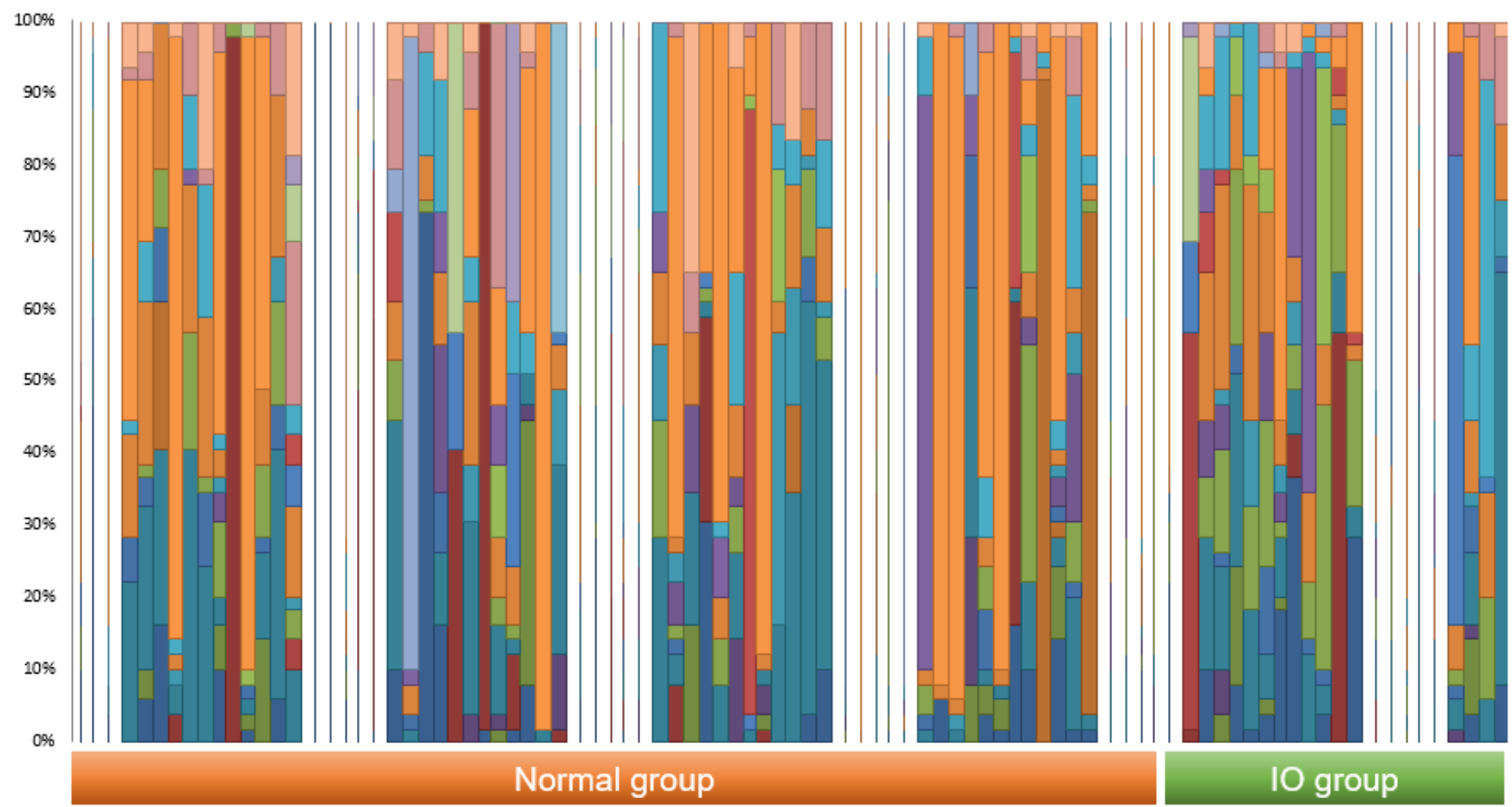

\section{Figure 1}

Taxon abundance in the vaginal microbiomes of study participants with normal and impaired ovarian (IO) function. 


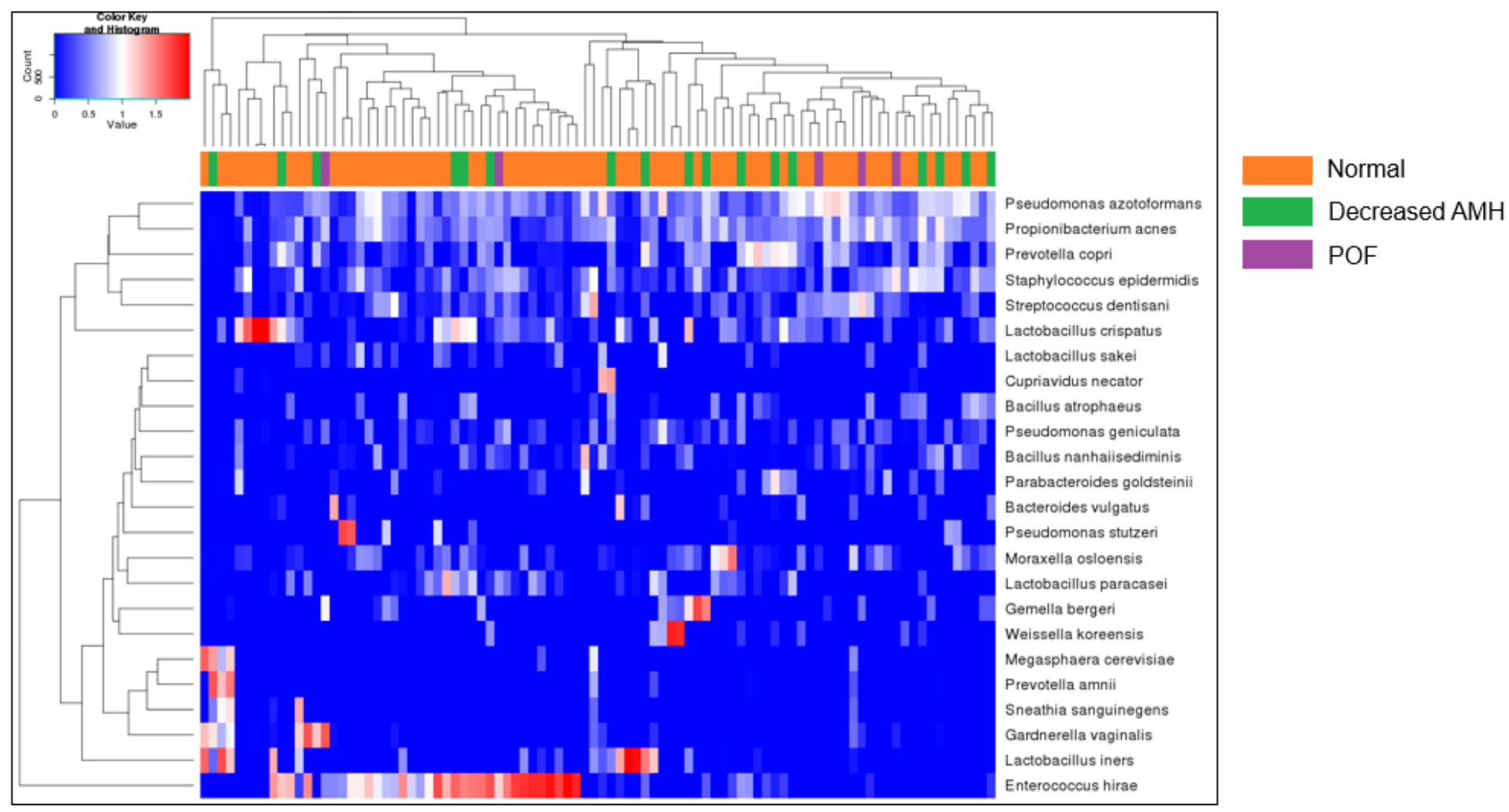

Figure 2

Heatmap of the vaginal microbiomes from study participants with normal and impaired ovarian function. 
Moraxella osloensis

Cupriavidus necator

Sneathia sanguinegens Megasphaera cerevisiae

Streptococcus dentisani

Weissella koreensis

Enterococcus hirae

Staphylococcus epidermidis

Gemella bergeri

Parabacteroides goldsteinii

Bacteroides vulgatus

Gardnerella vaginalis

Propionibacterium acnes

Bacillus nanhaiisediminis

Bacillus atrophaeus

Prevotella copri

Prevotella amnii

Pseudomonas geniculata

$P$ seudomonas stutzeri

Pseudomonas azotoformans

Lactobacillus sakei

Lactobacillus paracasei

Lactobacillus iners

Lactobacillus crispatus

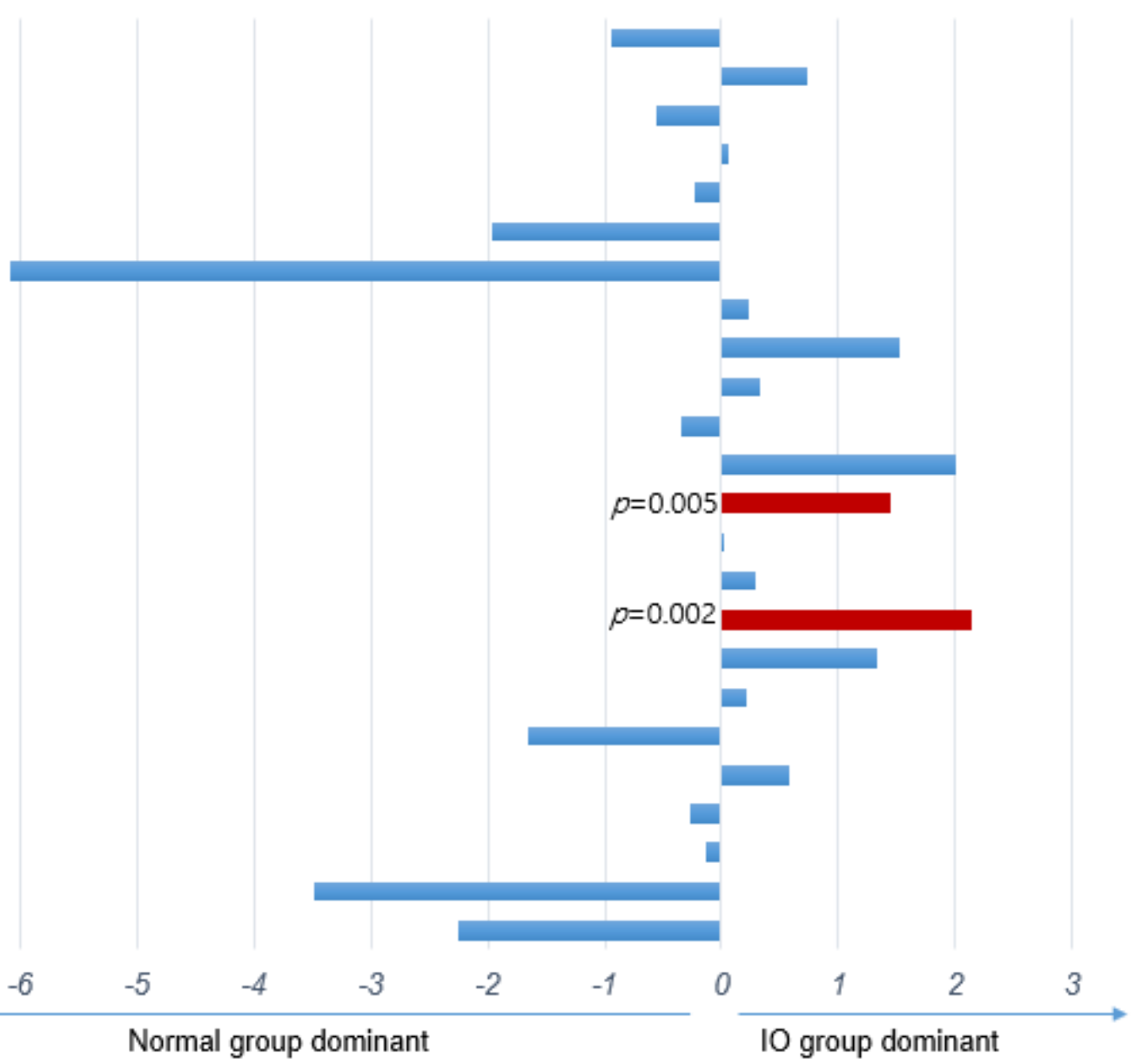

\section{Figure 3}

Differences in the dominant species in the vaginal microbiomes of the normal and impaired ovarian (IO) function groups.

(a) $2 \mathrm{D}$ plot

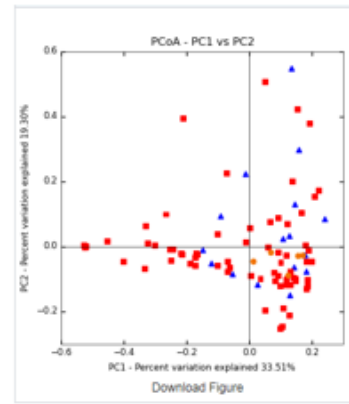

\begin{tabular}{lllll}
-7 & -6 & -5 & -4 & -3 \\
\hline & & & Normal group dominant
\end{tabular}

IO group dominant

(b) 3D plot
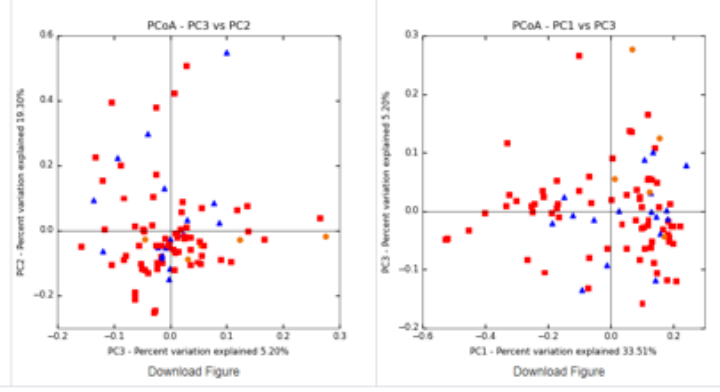

Normal group

Decreased AMH

POF

Figure 4

2D/3D plot and functional analyses of the vaginal microbiome. 


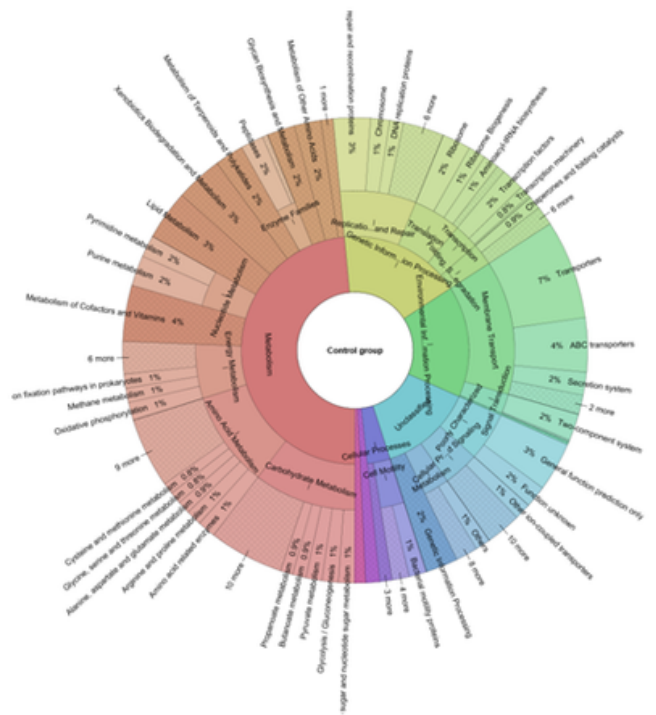

Normal ovarian function group

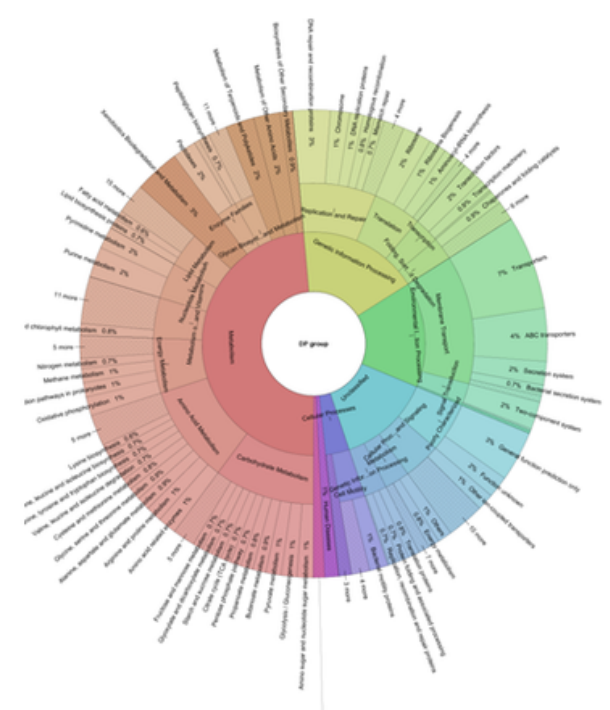

Impaired ovarian function group

\section{Figure 5}

2D/3D plot and functional analyses of the vaginal microbiome (a) 2D plot (b) 3D plot

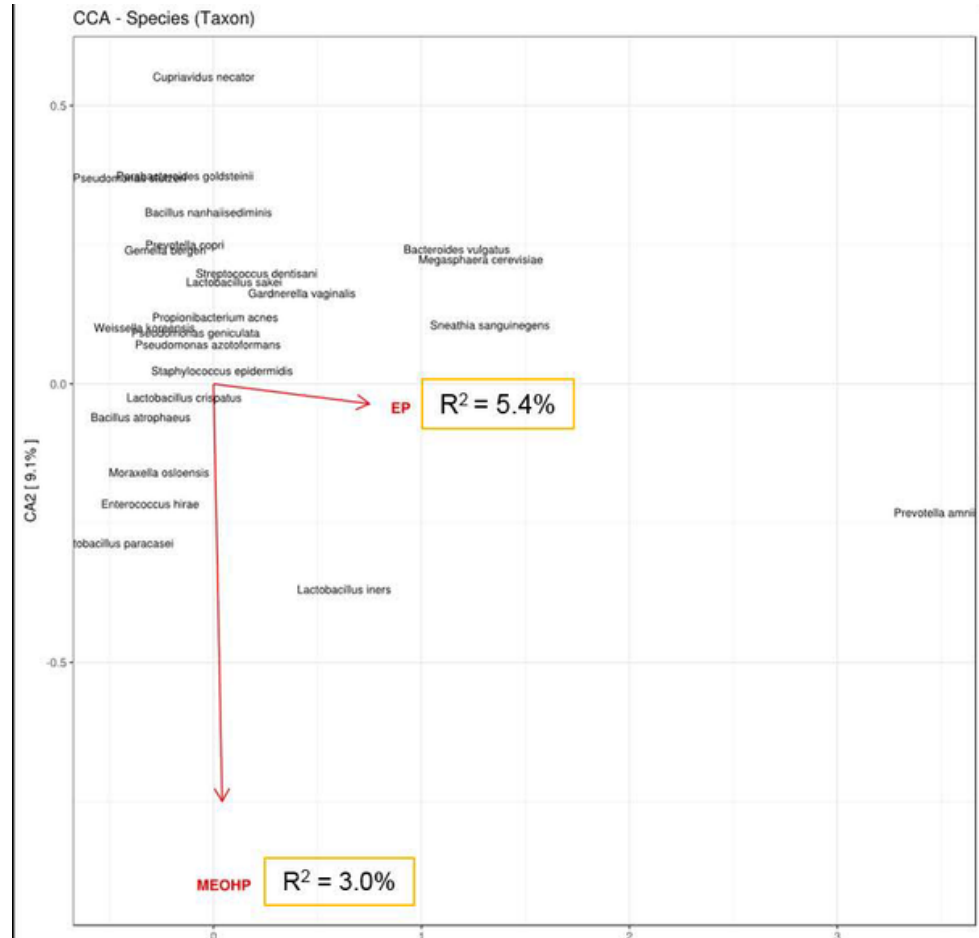

CA1 $[10.6 \%]$

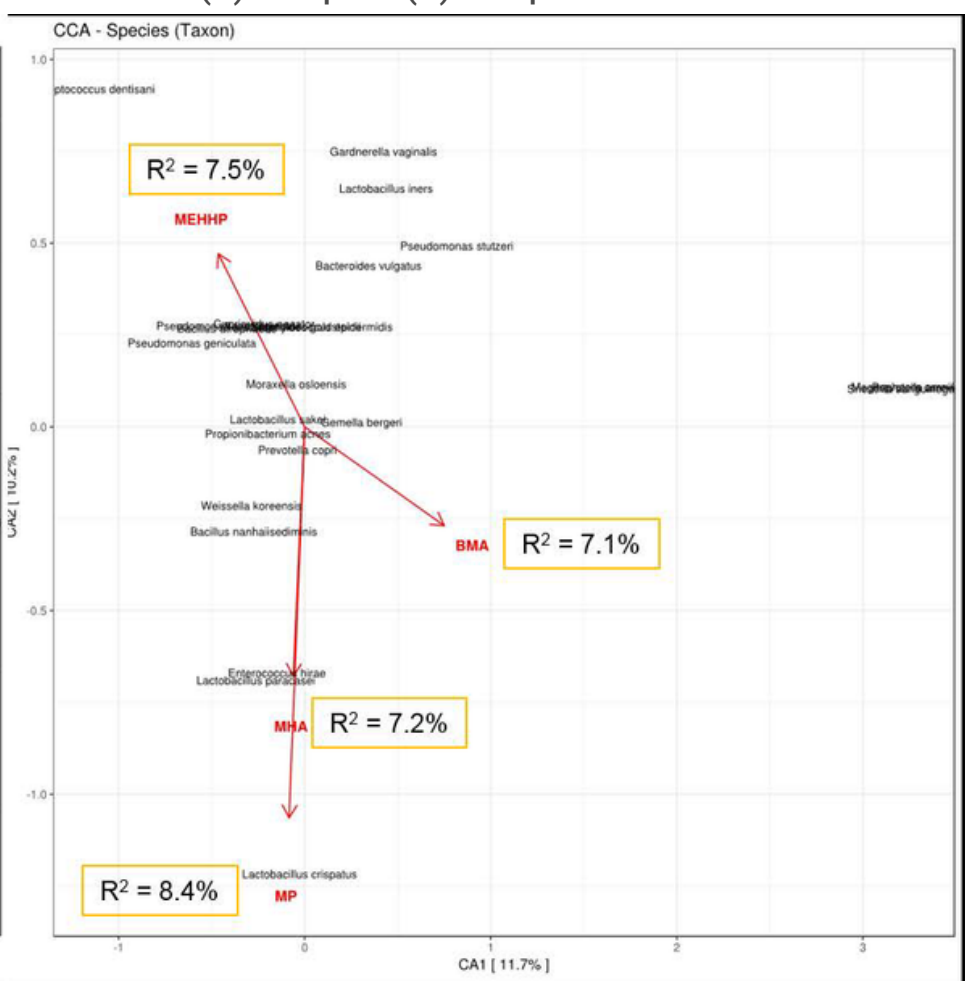

Impaired ovarian function group

Figure 6 
Canonical correlation analysis of the species in the normal and impaired ovarian function groups 